\title{
Serum Uric Acid as a Potential Biomarker in Patients With Rheumatoid Arthritis-associated Interstitial Pneumonia (RA-ILD)
}

\section{Zitao Wang}

Nanjing Medical University Second Affiliated Hospital

\section{Wen Wang}

Nanjing Medical University Second Affiliated Hospital

\section{Ting Xiang}

Nanjing Medical University Second Affiliated Hospital

\section{Bangdong Gong}

Tongji Hospital Affiliated to Tongji University: Shanghai Tongji Hospital

Jianmin Xie ( $\nabla$ xiejmdoc@163.com )

Department of Rheumatology, the Second Affiliated Hospital of Nanjing Medical University

\section{Research article}

Keywords: Rheumatoid arthritis, Uric acid, Interstitial lung disease, usual interstitial pneumonia pattern, EMT

Posted Date: July 19th, 2021

DOl: https://doi.org/10.21203/rs.3.rs-708089/v1

License: (c) (i) This work is licensed under a Creative Commons Attribution 4.0 International License. Read Full License 


\section{Abstract}

Background: Uric acid (UA) has been reported participate in various inflammatory and autoimmune diseases. Increasing evidence has shown that UA also plays an important role in lung inflammation and fibrosis. We aimed to investigate the correlation between UA and rheumatoid arthritis (RA), especially rheumatoid arthritis-associated interstitial lung disease (RA-ILD).

Methods: 266 RA patients and 138 healthy individuals were recruited in this study. RA was identified according to ACR/EULAR 2010 criteria. UA in serum and bronchoalveolar lavage fluid (BALF), as well as clinic and laboratory Indexes were collected from participants enrolled in the study. Serum KL-6 was measured via ELISA and then Spearman correlation analysis was used to analysing their association. Subsequent the receiver operating characteristic (ROC) curve and area under the curve (AUC) were used to estimate the diagnostic value of UA in RA-ILD and UIP pattern of RA-ILD. HRCT and pulmonary function tests (PFT) were performed to evaluated the characteristics and pulmonary findings of RA patients. Furtherly, we validated the effect of UA on the EMT and the expression of cytokines in A549 cells.

Results: Compared with healthy controls (HC), UA levels in serum was significantly higher in RA group $(262.25 \pm 91.94$ vs. $234.69 \pm 54.78 \mu \mathrm{mol} / \mathrm{L}, \mathrm{P}<0.01)$. Spearman correlation analysis revealed associations of SUA with risk factors of RA, including RF, anti-CCP, CRP and ESR $(r=0.37, p=0.02 ; r=0.48, p<0.01 ; r=0.26$, $p<0.01 ; r=0.27, p<0.01)$. And higher UA was measured both in serum ( $291.81 \pm 102.42$ vs.

$252.38 \pm 6.15 \mu \mathrm{mol} / \mathrm{L}, \mathrm{P}<0.01)$ and BALF $(393.3 \pm 222.6 \mathrm{vs} .204 .0 \pm 120.3 \mu \mathrm{mol} / \mathrm{L}, \mathrm{P}<0.01)$ of RA-ILD patients, particularly those with UIP pattern $(475.58 \pm 249.28$ vs. $262.86 \pm 103.45 \mu \mathrm{mol} / \mathrm{L}, p<0.01 ; 393.3 \pm 222.6$ vs. $204.0 \pm 120.3 \mu \mathrm{mol} / \mathrm{L}, \mathrm{p}<0.01)$. Meanwhile, the correlation between the level of UA in serum and BALF and serum KL-6 concentration in RA were also significant $(r=0.59, p<0.01 ; r=0.43, p<0.01)$. Also, the negative correlations of UA level, both in serum and BALF, with lung function parameters including FEV1/FVC and FEV1\% predicted were measured $(r=-0.78 ; r=-0.87, p<0.01)$. In the ROC curve analysis of AUC, the AUC value of UA was $0.76(95 \% \mathrm{Cl}=0.66-0.87, \mathrm{p}<0.01)$. The sensitivity and specificity were $60 \%$ and $92 \%$, respectively. The vitro experiment showed that UA stimulated the EMT in A549 cells, as well as induced the expression of cytokines, such as IL-1, IL- 6 and TGF- $\beta$, in lung epithelial cell.

Conclusions: This study suggests that UA is correlated with the ILD in RA. Particularly, the higher UA levels may be related to UIP, a pattern with worse prognosis, in patients with ILD. Therefore, UA may be an important contributing factor to the pathogenesis of RA-ILD.

\section{Introduction}

Uric acid (UA) is a final catabolite of purine found in organisms. In recent years, interest in uric acid as a regulator of inflammation and immune responses has grown[1, 2]. Previous publications suggested that UA has the ability to stimulate the expression of C-reactive protein (CRP), fibrinogen and complement C3 in a dose-dependent manner[3]. Growing evidence indicates that serum uric acid is closely associated with circulating levels of interleukin-6 (IL-6), CRP and tumor necrosis factor-a (TNF-a), which implies that 
UA may play a critical role in systemic inflammation and inflammatory-related diseases, such as rheumatoid arthritis (RA)[4]. Moreover, a series of studies have reported that uric acid crystals are not just innocent bystanders but may be active players in the proliferation and differentiation of $T$ cells that participate in the pathology of inflammatory disorders and autoinflammatory diseases. The experimental data demonstrated that UA coordinates with inflammasome-dependent cytokines to contribute to the differentiation of Th17 cells both in vitro and in vivo[5]. The correlation between Treg cell numbers and UA in systemic lupus erythematosus (SLE) has also been reported[6]. In addition, UA has been confirmed to be a considerable initiator and amplifier of Th2 cell function during immunity and allergic inflammation and to be an inflammatory mediator in allergic asthma[7]; additionally, it has been proposed that the local accumulation of UA, as an endogenous danger signal, can activate the Nod-like receptor protein 3 (NALP3) inflammasome, promoting the production of IL-1 3 , thereby contributing to lung inflammation and fibrosis[8]. Of note, increasing evidence has shown that a high level of UA, which is associated with both the presence of pulmonary hypertension $(\mathrm{PH})$ and higher mortality, has prognostic value in ILD[9]. Furthermore, recent studies verified that uric acid crystals formed at the lung injury site might represent a key danger signal for activating the inflammasome to release inflammatory cytokines, thereby causing inflammatory lung pathologies[8]. Moreover, increased UA serum concentrations in patients with lung disease of different etiologies correlate with the prognosis and severity of the disease[10, 11].

Rheumatoid arthritis (RA) is a chronic systemic inflammatory disease characterized by progressive and erosive destruction by arthritis accompanied by damage to multiple organs[12]. Interstitial lung disease (ILD), the most common extra-articular complication of RA, is associated with significant mortality and is a key factor affecting the prognosis of RA patients[13, 14]. Since RA-associated ILD (RA-ILD) has a high mortality rate, clarifying its pathogenesis and treatment strategies is necessary. Recently, some documents confirmed a novel role for the danger signal uric acid in driving proinflammatory Th17 differentiation, and significant relationships of systemic sclerosis (SSc) with pulmonary fibrosis and elevated UA levels have been reported[15]. Although the potential function of UA in lung disease has increasingly appreciated, there is little information available on the clinical significance of UA in RA patients with ILD.

Therefore, we hypothesize that UA might be engaged in the process of RA and is associated with the development of rheumatoid arthritis-associated interstitial lung disease (RA-ILD), and an assessment of UA may have clinical implications for identifying ILD with UIP patterns in RA patients. Herein, we analyzed the level of uric acid in RA-ILD patients and investigated the relationship between UA and other related laboratory indicators in RA-ILD patients. The data indicated that serum UA levels were correlated with the disease severity of RA-ILD patients, especially those with a UIP pattern.

\section{Materials And Methods}

Study population 
In our study, 266 patients (>18 years old) were recruited according to the 2010 ACR/EULAR criteria for definite RA at The Second Affiliated Hospital of Nanjing Medical University between January 2018 and December 2020. We excluded patients suffering from other autoimmune diseases, such as systemic lupus erythematosus (SLE) and dermatomyositis (DM), acute infectious disease, gout, and severe hepatic and renal dysfunction, or those taking uric acid-lowering drugs. Current or former smokers were also excluded. General condition, past medical history, smoking status and duration of RA were obtained from medical clinical records. The ILD of all patients collected in this study was defined according to HRCT evidence. Meanwhile, the patients were evaluated with pulmonary function tests (PFTs) and bronchoalveolar lavage (BAL). Additionally, 138 individuals matched by age and sex were also included as healthy controls (HCs).

All subjects who participated in this study voluntarily and provided written informed consent. The study protocol was approved by the medical ethics committee of The Second Affiliated Hospital of Nanjing Medical University.

Clinical and laboratory assessments

We registered the patients' general condition, including age, sex, duration of RA and comorbidities. Laboratory indexes such as C-reactive protein (CRP), erythrocyte sedimentation rate (ESR), presence and plasma level of rheumatoid factor (RF) and anti-cyclic peptide containing citrulline (anti-CCP) were collected. Serum samples of patients were analyzed for UA level and the concentration of Krebs von den Lungen-6 (KL-6). All basic demographic and clinical characteristics of the participants are shown in Table.

Bronchoalveolar lavage (BAL)

Based on current evidence, BAL cellular analysis might be a supplementary means for the diagnostic evaluation of ILD. All RA patients included in this study underwent BAL, bronchoalveolar lavage fluid (BALF) was collected and then UA concentrations were measured as described previously[16].

Measurement of Krebs von den Lungen-6 (KL-6)

The concentrations of KL- 6 in serum were measured by an enzyme-linked immunosorbent assay (ELISA) kit according to previously described procedures[17].

Radiographic classification

According to the American Thoracic Society/European Respiratory Society (ATS/ERS) classification of idiopathic interstitial pneumonias (IIPs) [18, 19], the HRCT patterns of RA-ILD were categorized into two major subgroups, namely, serum UAl interstitial pneumonia (UIP) and nonspecific interstitial pneumonia (NSIP). Separately, the UIP pattern was characterized by traction bronchiectasis/bronchiectasis, reticular opacity and honeycombing, while the HRCT characteristic of NSIP was extensive ground glass opacity[20]. HRCT scans of each patient were independently reviewed by two trained thoracic radiologists 
who were blinded to all clinical information such as demographics and general state. Disagreements over the diagnosis were resolved by consensus.

Pulmonary function tests (PFTs)

Patients with RA-ILD who underwent PFTs fulfilled the American Thoracic Society (ATS)/European Respiratory Society (ERS) guidelines[21-23], including forced vital capacity (FVC) and forced expiratory volume in $1 \mathrm{~s}$ (FEV1). All the data are presented as percentages of the normal predicted values. Moreover, we calculated the values of FEV1/FVC and FEV1\% predicted.

Cell culture, treatment and analysis

A549 cell line was used to resemble type II alveolar epithelial cells[24]. A549 cells were cultured in vitro. Before UA treatment, A549 cells were maintained in serum-free media for $12 \mathrm{~h}$ prior to stimulation and then changed to complete media with increasing concentrations of uric acid (UA, 0, 200 and $400 \mu \mathrm{mol} / \mathrm{L}$ ) for $24 \mathrm{~h}$. Supernatants were then collected and IL-1, IL-6, and TGF- $\beta$ concentrations were determined by ELISA.

Real time Reverse transcription semiquantitative polymerase chain reaction (Real-Time RT-PCR).

Total RNA was extracted from A549 cells by TRIzol ${ }^{\circ}$ RNA isolation (Gibco; Thermo Fisher Scientific, Inc.) and purified with DNase I (Invitrogen; Thermo Fisher Scientific, Inc.). according to the manufacturer's protocol. The primer sequences are available upon request. RNAs were reverse-transcribed into cDNA using SuperScriptTMII (Invitrogen Life Technology). Real-time quantitative PCR was performed by fluorescent dye SYBR Green methodology using SYBR Green PCR Master Mix (Applied Biosystems) and the ABI Prism 7000 apparatus (Perkin-Elmer, Foster City, CA). Gene expression was normalized to the corresponding $\beta$-actin level and is presented as the fold change relative to that of the control.

Immunofluorescence staining.

A549 cells were seeded in 6-well plates. After the indicated treatment, the cells were fixed and incubated with primary antibodies against a-SMA (Santa Cruz Biotechnology, Inc.) and E-cadherin (Santa Cruz Biotechnology, Inc.) overnight at $4^{\circ} \mathrm{C}$, after which the cells were washed three times with PBS. The cells were stained as described in the online Supplementary Information[25].

Statistical analysis

All statistical analyses were carried out using GraphPad Prism version 8.0.2. Differences between groups were analyzed by the Student's $t$-test. Comparisons of categorical variables were conducted using Pearson chi-square tests. For nonparametric data, the results were expressed as median (range) values, and the differences between groups were analyzed by the Mann-Whitney $U$ test. Spearman correlation analysis was performed to analyze the association of UA with clinical and laboratory indexes in RA patients. Univariate logistic regression analysis was performed to determine the factors associated with 
the presence of ILD. Multivariate logistic regression analysis was performed by including the confounding factors that were found to be significantly associated with the univariate analyses. The receiver operating characteristic (ROC) curve and area under the curve (AUC) were used to assess the validity and value of UA for RA-ILD. Any difference with a $p$ value $<0.05$ was regarded as statistically significant.

\section{Results}

\section{Relationship of serum UA level with laboratory indexes of RA patients}

The demographic characteristics, the UA level in serum and other laboratory values of RA patients are presented in Table 1. To investigate the relationship between the serum levels of UA and RA, we compared the demographics and serum UA level of the RA and control groups. The serum UA level in the RA patients was significantly higher than that in the healthy controls $(262.25 \pm 91.94$ vs. $234.69 \pm 54.78$ $\mu \mathrm{mol} / \mathrm{L}, \mathrm{P}<0.01$ ) (Fig. 1A). However, there was no significant difference in sex or age between the two groups. As shown in the Spearman correlation analysis, serum UA levels were positively correlated with RF and anti-CCP in patients with RA ( $r=0.37, p=0.02 ; r=0.48, p<0.01)$ (Fig. 2A, B). Next, we evaluated the strength of the association between serum UA and CRP and ESR. The results showed that the serum UA level was apparently correlated with CRP and ESR $(r=0.26, p<0.01 ; r=0.27, p<0.01)$.

\section{The UA level was elevated, especially in RA patients with ILD, and correlated with the KL-6 level}

Based on the above data of the RA and healthy groups, as well as the Spearman correlation analysis results, we concluded that UA may be an important predictor of RA. Furthermore, we explored whether the UA level was associated with RA-ILD in RA patients. The level of serum UA was significantly higher in patients with RA-ILD than in those without ILD $(291.81 \pm 102.42$ vs. $252.38 \pm 6.15 \mu \mathrm{mol} / \mathrm{L}, \mathrm{p}<0.01)$ (Fig. 1B). In addition, more UA was detected in the BALF of the RA-ILD group than in the BALF of the non-ILD group (393.3 \pm 222.6 vs. $204.0 \pm 120.3 \mu \mathrm{mol} / \mathrm{L}, \mathrm{p}<0.01$ ) (Fig. 1D). To further verify that UA levels were associated with lung injury markers in RA-ILD patients, we subsequently measured the concentration of $\mathrm{KL}-6$, a biomarker of ILDs[26], in the serum of RA patients and examined the relationship between the levels of UA and KL- 6 by correlation analysis. The results showed that serum UA levels positively correlated with KL-6 in patients with RA $(r=0.59, p<0.01)$ (Fig. 2C). Similarly, the UA level in BALF also had a powerful correlation with serum KL-6 in RA patients $(r=0.43, p<0.01)$ (Fig. 2D).

\section{High serum and BALF UA levels were correlated with the UIP pattern and the progression of RA-ILD}


The above data showed that the UA level was higher in RA patients with ILD than in patients without ILD, which meant that high UA levels may be implicated in the onset of ILD in RA. Clinically, RA-ILD manifests many well-recognized phenotypes, in which the usual interstitial pneumonia (UIP) pattern is the most common forms of RA-ILD with a worse prognosis[14]. Thus, the distinction of UIP in RA-ILD patients is of important clinical value for guiding the treatment of RA-ILD patients. In view of the aforementioned findings of UA levels in RA-ILD, we hypothesized that UA levels may be associated with the UIP pattern and the progression of RA-ILD. Based on the HRCT scans, 40 subjects with UIP patterns and 64 subjects with NSIP patterns were identified among the RA-ILD patients. Compared with the NSIP group, the level of serum UA in RA-ILD patients with the UIP pattern was markedly higher (475.58 \pm 249.28 vs. $262.86 \pm$ $103.45 \mu \mathrm{mol} / \mathrm{L}, \mathrm{p}<0.01$ ) (Fig. 1C). Interestingly, the UA level in BALF was significantly higher in RA-UIP patients than in RA-ILD patients with the NSIP pattern (393.3 \pm 222.6 vs. $204.0 \pm 120.3 \mu \mathrm{mol} / \mathrm{L}, \mathrm{p}<0.01$ ) (Fig. 1E). To investigate whether the UA level was correlated with a decline in lung function in RA-UIP patients, we evaluated the lung function of RA-ILD patients with the UIP and NSIP patterns. The results showed that the UA levels in serum and BALF were negatively correlated with FEV1/FVC $(r=-0.78, P<0.01$; $r=-0.46, P<0.01)$ (Fig. 3A, C) and FEV1\% predicted ( $r=-0.87, p<0.01 ; r=-0.47, p<0.01)$ (Fig. 3B, D). These data suggest that UA may be a significant contributor to the pathogenesis of RA-ILD.

\section{Serum UA levels can be a disease marker for the detection of RA-ILD with UIP}

To evaluate the diagnostic value of the serum UA level in clinical settings, we established an ROC curve and examined the sensitivity and specificity of the circulating factors in identified RA patients with UIP. The area under the curve (AUC) was 0.661 (95\% Cl: 0.542-0.780; $p<0.001$ ), with a sensitivity and specificity of $51.7 \%$ and $79.3 \%$, respectively. (Fig. 3E). Thus, these results suggest that serum UA may be a positive biomarker for distinguishing the UIP pattern in RA-ILD patients, meaning that it can be a significant biomarker candidate for appraising the disease progression and severity of ILD in RA patients.

\section{UA induces epithelial-to-mesenchymal transition (EMT) in A549 cells}

Emerging evidence has demonstrated that EMT phenomena are potential contributors to ILD, that alveolar epithelial cells from interstitial lung diseases undergo epithelial-to-mesenchymal transitions after lung injury, and that these alveolar epithelial cells undergo EMT, which includes loss of their epithelial biomarkers and acquisition of mesenchymal (fibroblast-like) cell biomarkers[27]. To assess whether UA could induce EMT in A549 cells, we performed immunofluorescence staining of EMT markers, including E-cadherin and $\mathbf{a}$-SMA, after A549 cells were incubated with UA (0,200 and $400 \mu \mathrm{M})$ for $24 \mathrm{~h}$. We found that UA decreased the expression of the epithelial cell marker E-cadherin in a dose-dependent manner(Fig. $4 A, B)$. In contrast, UA augmented the expression of the mesenchymal cell marker $\mathbf{a}-S M A$ in a dosedependent manner(Fig. 4C, D). These findings revealed that high UA was shown to induce EMT in A549 cells. 


\section{The production of cytokines was induced by high UA in A549 cells}

It has been demonstrated that these proinflammatory cytokines exert direct proinflammatory effects on alveolar and bronchial epithelial cells, which are well-known lung pathology promoters[28, 29]. Next, we explored whether UA is capable of stimulating the release of cytokines from A549 cells. A549 cells were treated with increasing concentrations of $U A(0,200$ and $400 \mu \mathrm{M})$, and supernatants of A549 cells stimulated with UA were harvested for ELISA analysis. As shown in Fig. 5A.B.C, the production of IL-1, IL-6 and transforming growth factor- $\beta$ (TGF- $\beta$ ) by A549 alveolar epithelial cells was markedly increased by UA in a dose-dependent manner compared with that of the control group, both at $24 \mathrm{~h}$ after treatment. A549 cells treated with increasing concentrations of UA were also harvested for quantitative RT-PCR analysis. As shown in Fig. 5D.E.F, the mRNA expression of IL-1, IL-6 and TGF- $\beta$ was upregulated by UA in a dosedependent manner compared with that of the control group, both at $24 \mathrm{~h}$ after treatment. These results revealed that high UA could induce the expression of cytokines in A549 cells.

\section{Discussion}

This is the first report on the strong association between serum UA levels and the severity and progression of UIP in RA patients with ILD relative to RA patients without ILD. Furthermore, serum UA levels were significantly increased in the UIP pattern of RA-ILD patients. Intriguingly, the increased serum UA level in RA-ILD patients was positively correlated with the HRCT-UIP score (Fig. 2E) and rheumatoid factor (RF). ROC curves also showed that the serum UA level was an independent biomarker for the identification of disease severity and UIP in RA patients. These data suggest that the circulating UA concentration may be a potential biomarker for identifying UIP and evaluating the progression and severity of ILD in RA patients.

UA is an antioxidant that is remarkable in the airway mucous membrane, and its actual role, especially in the regulation of lung function, has attracted attention in various diseases[16, 30]. The present study showed that the level of UA was significantly associated with RA and the presence of ILD in RA, and a higher UA level was measured in the serum of individuals with RA. Significant correlations between serum UA levels and other related laboratory parameters in RA, including RF and anti-CCP, were observed. Additionally, compared with RA patients without ILD, the levels of serum and BALF UA were robustly higher in patients with RA-ILD, suggesting that UA may have specific correlations with ILD in RA patients. Furthermore, the serum UA level was elevated in the UIP pattern of RA-ILD patients compared to the NSIP pattern of RA-ILD patients, which indicated the apparent correlation between UA and the severity of ILD in RA. Thus, we believe that UA plays an important role in the pathogenesis of RA-ILD.

Regarding the underlying work, we have confirmed that serum UA levels have an obvious association with RA-ILD, and circulating UA levels have a strong correlation with ILD structural damage (HRCT score) and a significant correlation with pulmonary function impairment (FEV1/FVC, FEV1). Most notably, the circulating UA level has a remarkable association with the clinically severest RA-ILD disease phenotype, 
i.e., the UIP pattern. The overall function of UA in terms of its association with ILD is apparently correlated with the currently available marker KL-6. The good predictability of the serum UA level implied a potential role as a new biomarker for RA-ILD.

Some experimental and clinical reports have confirmed that UA might be closely related to inflammatory responses, and serum UA levels are positively associated with TNF-a, IL-6 and CRP[16, 31]. Interestingly, the results of an in vitro experimental study revealed that monosodium urate crystals can activate human fibroblast-like synoviocytes (FLSs) from RA patients with a remarkable increase in the release of the inflammatory cytokine IL-6, which provided insight into the role of UA in provoking inflammation in RA[32]. In our study, the level of serum UA showed significant positive correlations with several inflammatory risk factors for RA, including CRP and ESR, suggesting that serum UA levels may be used as an effective indicator for predicting the inflammatory activity of RA.

Interstitial lung diseases are characterized by destruction of lung architecture due to excessive deposition of extracellular matrix proteins by myofibroblast activation, leading to aggravated dyspnea and loss of lung function[33,34]. Epithelial-mesenchymal transition (EMT) plays a critical role in the accumulation of myofibroblasts and the resultant deposition of extracellular matrix proteins, which is a crucial step in the progression of ILD[35]. Both EMT and aberrant phenotypic changes in alveolar epithelial cells are prominent for the progression of ILD $[33,36]$. To further verify that UA is involved in the pathogenesis of ILD, we investigated whether UA contributed to EMT of alveolar epithelial cells. Our data revealed that UA could significantly induce EMT in alveolar epithelial cells (A549 cells), including elevation of mesenchymal markers and decline of epithelial cell markers. In a recent study, soluble UA was found to contribute to lung interstitial fibrosis closely related to the NLRP3 inflammatory pathway[8]. In contrast, the consensus among the previous works showed that some proinflammatory cytokines, such as IL-1, IL6 and TGF- $\beta$, secreted by alveolar epithelial cells are closely related to the development of ILD[37]. Thus, we detected the expression of IL-1, IL-6, and TGF- $\beta$ in A549 cells treated with different concentrations of UA, and the results indicated that IL-1, IL-6, and TGF- $\beta$ expression was elevated by UA in a dosedependent manner, suggesting the considerable value of UA in ILD pathological processes.

\section{Conclusions}

In conclusion, serum UA levels were significantly increased in the UIP pattern of RA-ILD patients. Intriguingly, the increased serum UA level in RA-ILD patients with UIP was positively correlated with the HRCT-score, RF and anti-CCP. ROC curves also showed that the serum UA level was an independent biomarker for the identification of disease severity and UIP in RA patients. Moreover, the vitro experiment further showed that UA induced epithelial-to-mesenchymal transition (EMT) and production of IL-1, IL-6 and TGF- $\beta$ in A549 cells. Thus, this study established that UA is strongly associated with RA-ILD, especially UIP pattern, which can be an important indicator for predicting the onset and severity of RAILD. UA may also hold promise as a useful biomarker for RA-ILD.

\section{Abbreviations}


CRP: Creactive protein; IL-1: Interleukin-1; IL-6: Interleukin-6; IL-8: Interleukin-8; ILD: Interstitial lung disease; RF: rheumatoid factor; anti-CCP: anti-cyclic peptide containing citrulline; ESR: erythrocyte sedimentation rate; UA: uric acid; KL-6: Krebs von den Lungen-6; NSAIDs: Nonsteroidal Antiinflammatory Drugs; DMARDs: disease-modifying anti-rheumatic drugs; DAS 28: 28-joint Disease Activity Score.

\section{Declarations}

\section{Ethics approval and consent to participate}

All patients gave written informed consent before any study-related procedures were performed. The study protocol was approved by the local institutional ethics committee of Nanjing Medical University.

\section{Acknowledgements}

We are grateful to all the patients who took part in this study as well as our colleagues at the Institute of Rheumatology for their help in recruiting patients for the study.

\section{Authors' contributions}

Study conception and design: XJM and WZT; acquisition of data: WZT; WW and XT; Analysis and interpretation of data: XJM, GBD, WZT, WW and XT. All authors were involved in drafting the manuscript or revising it critically for content. All the authors approved the final version for publication.

\section{Funding}

This study was supported by grants from the National Natural Science Foundation of China (No. 81871279), The funders had no role in study design, data collection and analysis, decision to publish, or preparation of the manuscript.

\section{Availability of data and materials}

The datasets used and/or analyzed during the current study are available from the corresponding author on reasonable request.

\section{Consent for publication}

Not applicable.

\section{Competing interests}

The authors declare that they have no competing interests

\section{References}


1. Neumann $\mathrm{K}$, Castiñeiras-Vilariño $\mathrm{M}$, Höckendorf $\mathrm{U}$, et al. Clec12a is an inhibitory receptor for uric acid crystals that regulates inflammation in response to cell death. Immunity 2014;40:389-99.

2. Ghaemi-Oskouie F, Shi Y. The role of uric acid as an endogenous danger signal in immunity and inflammation. Curr Rheumatol Rep 2011;13:160-6.

3. Spiga R, Marini MA, Mancuso E, et al. Uric acid is associated with inflammatory biomarkers and induces inflammation via activating the nf-kappab signaling pathway in hepg2 cells. Arterioscler Thromb Vasc Biol 2017;37:1241-9.

4. Lyngdoh T, Marques-Vidal P, Paccaud F, et al. Elevated serum uric acid is associated with high circulating inflammatory cytokines in the population-based colaus study. PLoS One 2011;6:e19901.

5. Conforti-Andreoni C, Spreafico R, Qian HL, et al. Uric acid-driven th17 differentiation requires inflammasome-derived il-1 and il-18. 2011;187:5842-50.

6. Huang $\mathrm{H}$, Sun $\mathrm{W}$, Liang $\mathrm{Y}$, et al. Treg cells in thrombotic thrombocytopenic purpura associated with systemic lupus erythematosus patients. Renal Failure 2014;36:1263-7.

7. Kool M, Willart MA, van Nimwegen $M$, et al. An unexpected role for uric acid as an inducer of thelper 2 cell immunity to inhaled antigens and inflammatory mediator of allergic asthma. Immunity 2011;34:527-40.

8. Gasse $\mathrm{P}$, Riteau N, Charron S, et al. Uric acid is a danger signal activating nalp3 inflammasome in lung injury inflammation and fibrosis. Am J Respir Crit Care Med 2009;179:903-13.

9. Seyyed Reza Seyyedi MM, Mandana Chitsazan , Neda Behzadnia , Makan Sadr, Seyed Mohammadreza Hashemian , Babak SharifKashani Relationship between serum uric acid levels and the severity of pulmonary hypertension. National Research Institute of Tuberculosis and Lung Disease 2017

10. Rumora L, Hlapcic I, Popovic-Grle S, Rako I, Rogic D, Cepelak I. Uric acid and uric acid to creatinine ratio in the assessment of chronic obstructive pulmonary disease: Potential biomarkers in multicomponent models comprising il-1beta. PLoS One 2020;15:e0234363.

11. Dhaun N, Vachiery JL, Benza RL, et al. Endothelin antagonism and uric acid levels in pulmonary arterial hypertension: Clinical associations. J Heart Lung Transplant 2014;33:521-7.

12. Juge PA, Crestani $B$, Dieude P. Recent advances in rheumatoid arthritis-associated interstitial lung disease. Curr Opin Pulm Med 2020;26:477-86.

13. Rojas-Serrano J, Herrera-Bringas D, Perez-Roman DI, Perez-Dorame R, Mateos-Toledo H, Mejia M. Rheumatoid arthritis-related interstitial lung disease (ra-ild): Methotrexate and the severity of lung disease are associated to prognosis. Clin Rheumatol 2017;36:1493-500.

14. Spagnolo P, Lee JS, Sverzellati N, Rossi G, Cottin V. The lung in rheumatoid arthritis: Focus on interstitial lung disease. Arthritis Rheumatol 2018;70:1544-54.

15. Dimitroulas $T$, Giannakoulas $G$, Dimitroula $H$, et al. Significance of serum uric acid in pulmonary hypertension due to systemic sclerosis: A pilot study. Rheumatol Int 2011;31:263-7. 
16. Kobayashi T, Nakagome K, Noguchi T, et al. Elevated uric acid and adenosine triphosphate concentrations in bronchoalveolar lavage fluid of eosinophilic pneumonia. Allergol Int 2017;66S:S27S34.

17. TAKAHASHI T, MUNAKATA M, SUZUKI I, KAWAKAMI Y. Serum and bronchoalveolar fluid kl-6 levels in patients with pulmonary alveolar proteinosis. 1998;158:1294-8.

18. American thoracic society. Idiopathic pulmonary fibrosis: Diagnosis and treatment. International consensus statement. $\mathrm{T}$ his $\mathrm{j}$ oint $\mathrm{s}$ tatement of the a merican t horacic s ociety (ats), and the $\mathrm{e}$ uropean r espiratory s ociety (ers) was a dopted by the ats b oard of $d$ irectors , j uly 1999 and by the ers e xecutive c ommittee , o ctober 1999. Am J Respir Crit Care Med 2000;161:646-64.

19. Travis WD, Costabel U, Hansell DM, et al. An official american thoracic society/european respiratory society statement: Update of the international multidisciplinary classification of the idiopathic interstitial pneumonias. Am J Respir Crit Care Med 2013;188:733-48.

20. Raghu G, Remy-Jardin M, Myers JL, et al. Diagnosis of idiopathic pulmonary fibrosis. An official ats/ers/jrs/alat clinical practice guideline. Am J Respir Crit Care Med 2018;198:e44-e68.

21. Wanger J, Clausen JL, Coates A, et al. Standardisation of the measurement of lung volumes. Eur Respir J 2005;26:511-22.

22. Miller MR, Hankinson J, Brusasco V, et al. Standardisation of spirometry. Eur Respir J 2005;26:31938.

23. Macintyre N, Crapo RO, Viegi G, et al. Standardisation of the single-breath determination of carbon monoxide uptake in the lung. Eur Respir J 2005;26:720-35.

24. Lieber M, Smith B, Szakal A, Nelson-Rees W, Todaro G. A continuous tumor-cell line from a human lung carcinoma with properties of type ii alveolar epithelial cells. Int J Cancer 1976;17:62-70.

25. Donaldson JG. Immunofluorescence staining. Current protocols in cell biology 2015;69:4.3.1-4.3.7.

26. Lee JS, Lee EY, Ha YJ, Kang EH, Lee YJ, Song YW. Serum kl-6 levels reflect the severity of interstitial lung disease associated with connective tissue disease. Arthritis Res Ther 2019;21:58.

27. Kage H, Borok Z. Emt and interstitial lung disease: A mysterious relationship. Curr Opin Pulm Med 2012;18:517-23.

28. Chen Z, Bozec A, Ramming A, Schett G. Anti-inflammatory and immune-regulatory cytokines in rheumatoid arthritis. Nat Rev Rheumatol 2019;15:9-17.

29. Buckley ST, Medina C, Ehrhardt C. Differential susceptibility to epithelial-mesenchymal transition (emt) of alveolar, bronchial and intestinal epithelial cells in vitro and the effect of angiotensin ii receptor inhibition. Cell and tissue research 2010;342:39-51.

30. Wattanachayakul P, Rujirachun P, Charoenngam N, Ungprasert P. Chronic obstructive pulmonary disease (copd) is associated with a higher level of serum uric acid. A systematic review and metaanalysis. Adv Respir Med 2020;88:215-22.

31. Yan Shi ADM, Gilbert Ng Monosodium urate crystals in inflammation and immunity. Immunological Reviews 2009;233: 203-217 
32. Chen DP, Wong CK, Tam LS, Li EK, Lam CW. Activation of human fibroblast-like synoviocytes by uric acid crystals in rheumatoid arthritis. Cell Mol Immunol 2011;8:469-78.

33. Wolters PJ, Collard HR, Jones KD. Pathogenesis of idiopathic pulmonary fibrosis. Annu Rev Pathol 2014;9:157-79.

34. Richeldi L, Collard HR, Jones MG. Idiopathic pulmonary fibrosis. The Lancet 2017;389:1941-52.

35. Marmai C, Sutherland RE, Kim KK, et al. Alveolar epithelial cells express mesenchymal proteins in patients with idiopathic pulmonary fibrosis. Am J Physiol Lung Cell Mol Physiol 2011;301:L71-8.

36. Kim KK, Kugler Mc Fau - Wolters PJ, Wolters Pj Fau - Robillard L, et al. Alveolar epithelial cell mesenchymal transition develops in vivo during pulmonary fibrosis and is regulated by the extracellular matrix. PNAS 2006;103

37. Hetzel M, Bachem M, Anders D, Trischler G, Faehling M. Different effects of growth factors on proliferation and matrix production of normal and fibrotic human lung fibroblasts. Lung 2005;183:225-37.

\section{Table}

Table 1 Demographic, clinical and laboratory characteristics of RA-ILD patients $(n=98)$ 


\begin{tabular}{|c|c|c|c|}
\hline Characteristics & $\operatorname{NSIP}(n=54)$ & UIP $(n=44)$ & $\mathrm{p}$-value \\
\hline \multicolumn{4}{|l|}{ General demographics } \\
\hline Sex (male/female)(\%female) & $15(27.8)$ & 13(29.6) & 0.781 \\
\hline Age (mean $\pm S D$, years) & $52.44 \pm 2.73$ & $57.18 \pm 3.56$ & 0.462 \\
\hline Duration of the disease (months) & $63(6-360)$ & $62(1-360)$ & 0.581 \\
\hline \multicolumn{4}{|l|}{ Laboratory values } \\
\hline RF (IU/ml) & $156.5 \pm 81.2$ & $324.5 \pm 220.8$ & 0.009 \\
\hline anti-CCP $\otimes R U / m l \rrbracket$ & $367.3 \pm 235.6$ & $581.6 \pm 462.7$ & 0.004 \\
\hline $\operatorname{ESR}(\mathrm{mm} / \mathrm{h})$ & $48.27 \pm 6.74$ & $52.61 \pm 9.64$ & 0.367 \\
\hline $\mathrm{CRP} \rrbracket \mathrm{mg} / \mathrm{L} \otimes$ & $46.75 \pm 4.85$ & $81.39 \pm 15.49$ & 0.036 \\
\hline $\mathrm{UA}(\mu \mathrm{mol} / \mathrm{L})$ & $262.86 \pm 103.45$ & $475.58 \pm 249.28$ & 0.001 \\
\hline $\mathrm{KL}-6$ (U/ml) & $778.86 \pm 689.51$ & $1058.53 \pm 1168.55$ & 0.003 \\
\hline \multicolumn{4}{|l|}{ Bronchoalveolar lavage (BAL) } \\
\hline $\mathrm{UA}(\mu \mathrm{mol} / \mathrm{L})$ & $204.0 \pm 120.3$ & $393.3 \pm 222.6$ & 0.008 \\
\hline \multicolumn{4}{|l|}{ Current treatments } \\
\hline NSAIDS & 18(33.3) & $11(25.0)$ & 0.471 \\
\hline Glucocorticoids & $23(42.6)$ & $16(36,4)$ & 0.168 \\
\hline MTX & $17(31.5)$ & $12(27.3)$ & 0.652 \\
\hline bioDMARDs & $15(27.8)$ & $9(20.4)$ & 0.536 \\
\hline \multicolumn{4}{|l|}{ Clinical features } \\
\hline RA duration(mean \pm SD) (yrs) & $5.46 \pm 2.62$ & $9.71 \pm 5.28$ & 0.153 \\
\hline DAS28 score & $6.45 \pm 1.25$ & $6.82 \pm 2.58$ & 0.421 \\
\hline Cough & $31(61.1)$ & $24(54.6)$ & 0.732 \\
\hline Sputum & $17(31.5)$ & $10(22.7)$ & 0.652 \\
\hline Dyspnea & $15(27.8)$ & $21(47.8)$ & 0.274 \\
\hline Chest congestion & 11(20.3) & $17(38.6)$ & 0.561 \\
\hline Dry rale & 17(31.5) & $21(47.7)$ & 0.235 \\
\hline Bluish skin tinge & $2(3.70)$ & $1(2.27)$ & 0.851 \\
\hline Clubbing & $3(5.56)$ & $0(0)$ & 0.257 \\
\hline
\end{tabular}


RF: rheumatoid factor; anti-CCP: anti-cyclic peptide containing citrulline; ESR: erythrocyte sedimentation rate; CRP: C-reactive protein; UA: uric acid; KL-6: Krebs von den Lungen-6; NSAIDs: Nonsteroidal Antiinflammatory Drugs; DMARDs: disease-modifying anti-rheumatic drugs; DAS 28: 28-joint Disease Activity Score.

\section{Figures}
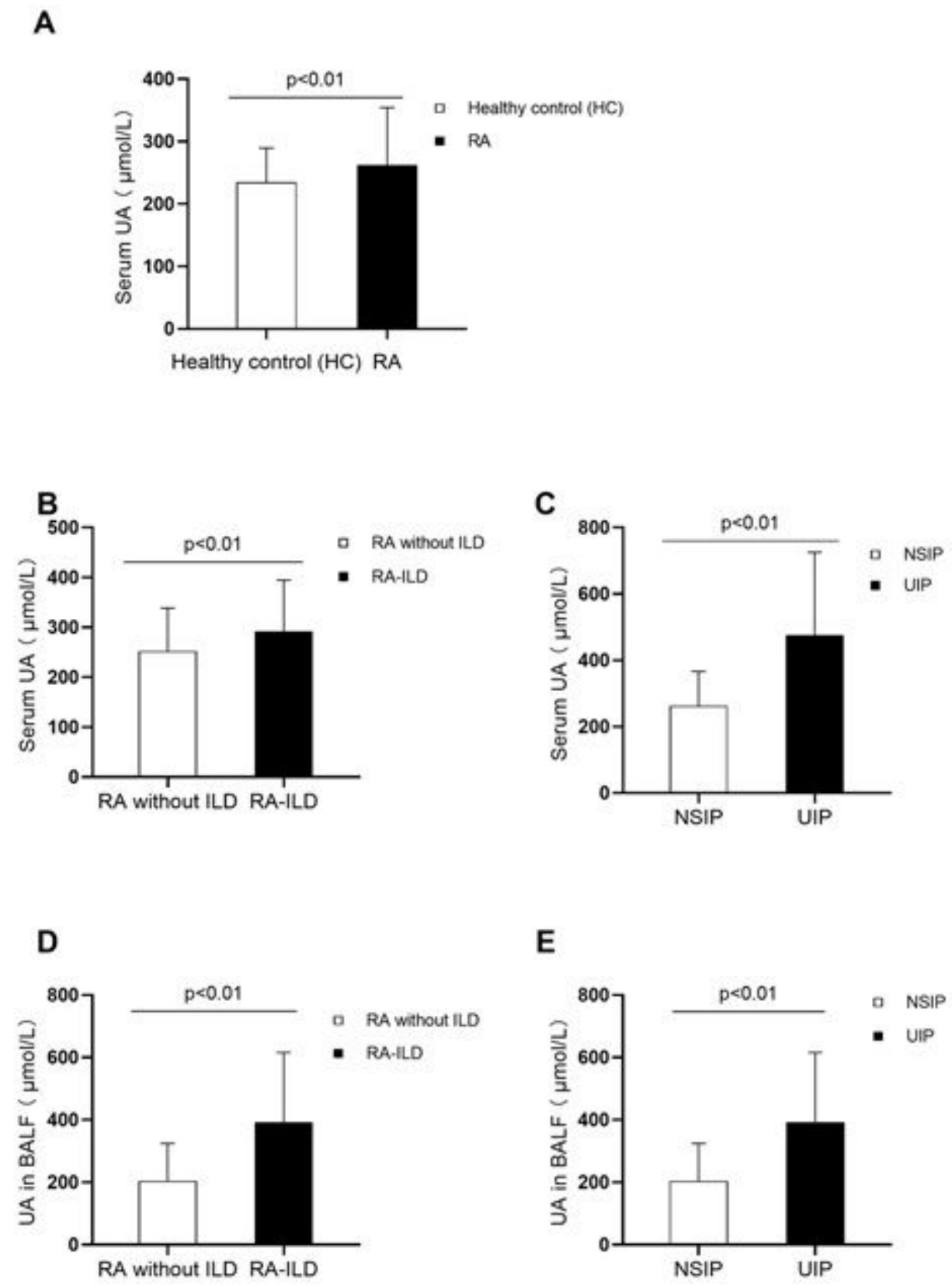

Figure 1

The level of UA was measured in RA-ILD patients. The level of UA in serum of RA patients and healthy control $(H C)$. Statistical difference was detected between RA patients and $H C(p<0.01)(A)$. Comparison of serum UA levels between no-ILD and RA-ILD, as well as NSIP and UIP. The higher serum UA levels were 
observed in RA-ILD relative to RA without ILD ( $p<0.01)(B)$. Statistical difference of serum UA was also detected between UIP and NSIP pattern of RA-ILD $(p<0.01)(C)$. The UA level in BALF of RA patients was also measured. Compared with RA patients without ILD, the level of UA in RA-ILD patients was higher significantly $(p<0.01)(D)$. And a marked increase of the level of UA was observed in BALF in RA-UIP patients relative to RA patients with NSIP pattern $(p<0.01)(E)$.

A
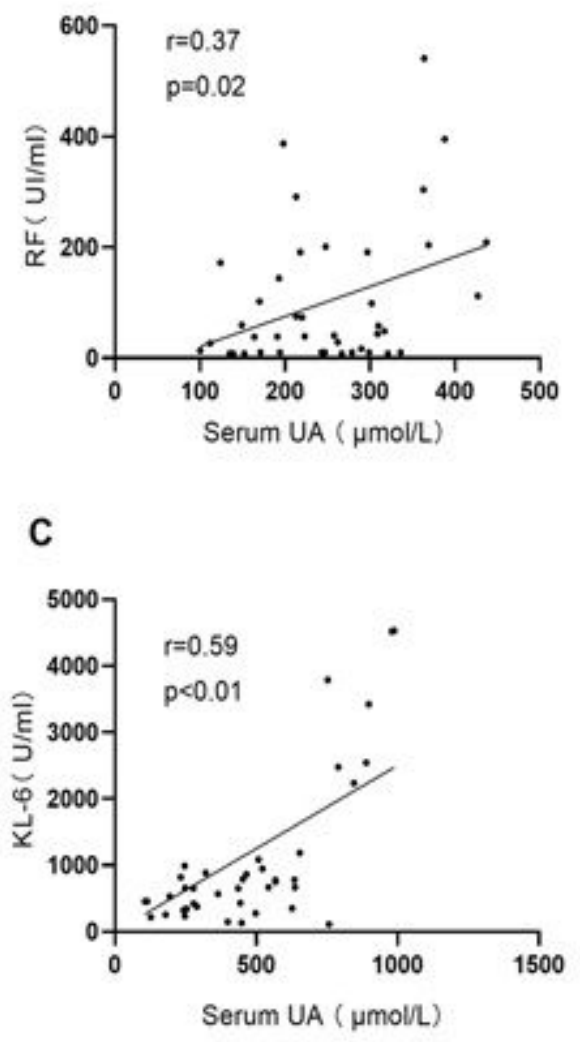

$E$

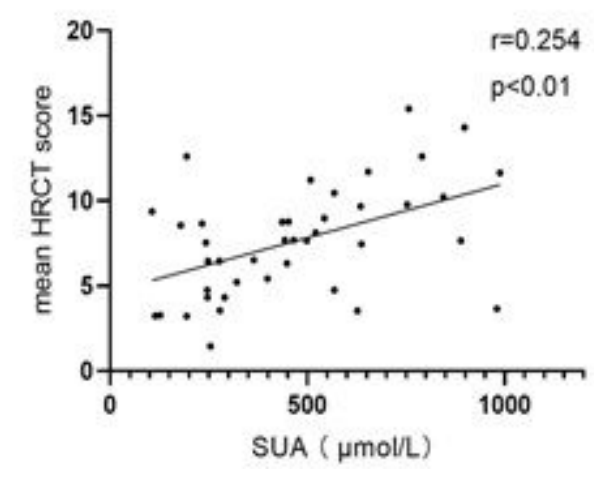

B

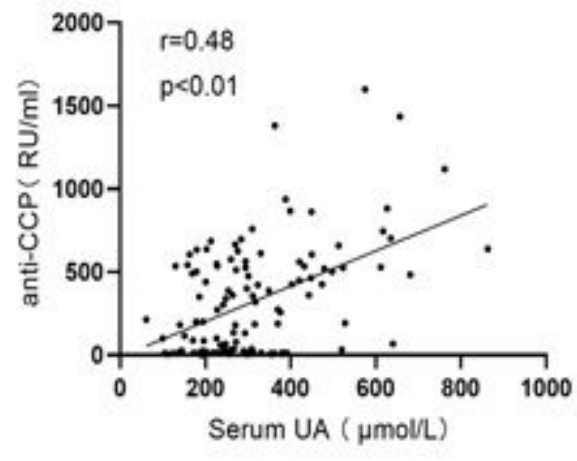

D

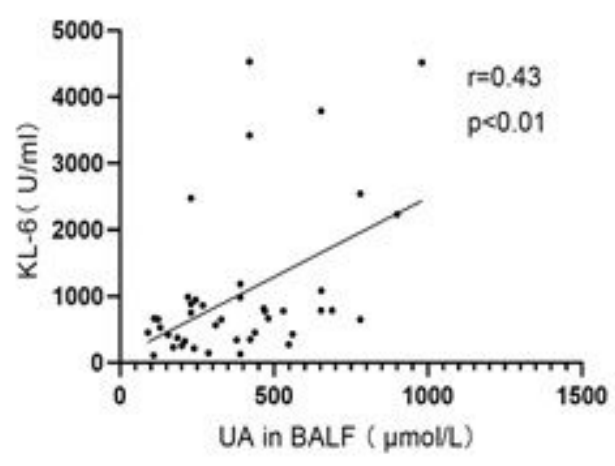

Figure 2

Correlation of UA with clinic and laboratory Indexes in RA patients. RF and anti-CCP were positively correlated with the serum UA in patients with RA (A-B). A positive correlation between serum UA and KL- 6 was detected by Spearman test (C). UA in BALF was positively associated with KL-6 in RA (D). Serum UA also showed a positive correlation with the mean HRCT score (E). 
A

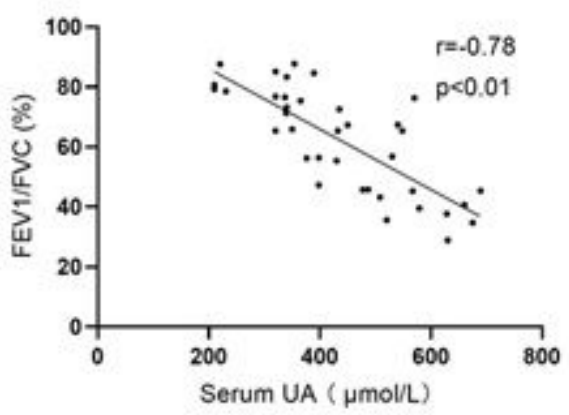

C

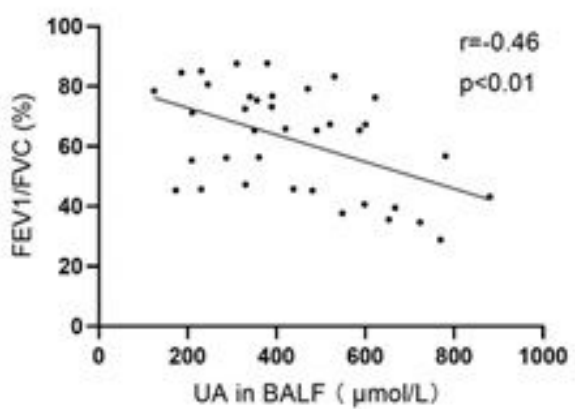

E

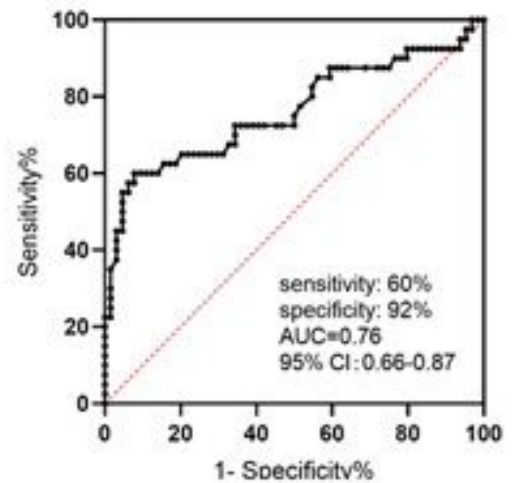

B

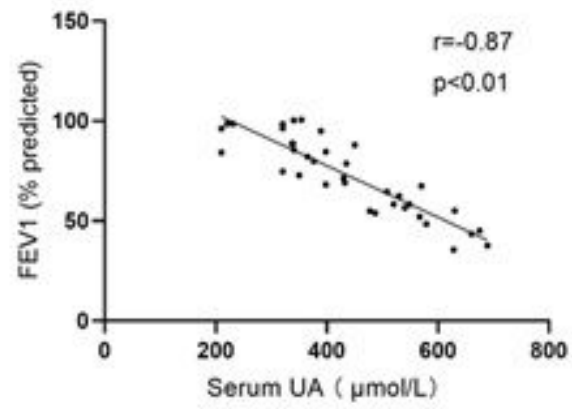

D

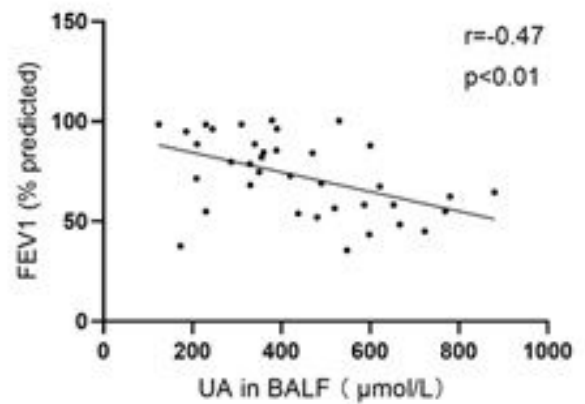

\section{Figure 3}

Correlations between the UA level in serum and BALF and lung function. Serum UA levels were inversely correlated with FEV1/FVC (A) and FEV1\% predicted (B) in RA-ILD patients. And UA levels in BALF negatively correlated with pulmonary function indexes (C-D). Receiver operating characteristic (ROC) curve showing the predictive capacity of UA in the presence of UIP pattern in RA. The area under the ROC curve (AUC) was 0.76 (95\% Cl=0.66-0.87; sensitivity: 60\%; specificity: 92\%; $p<0.01)$ (E). 
A
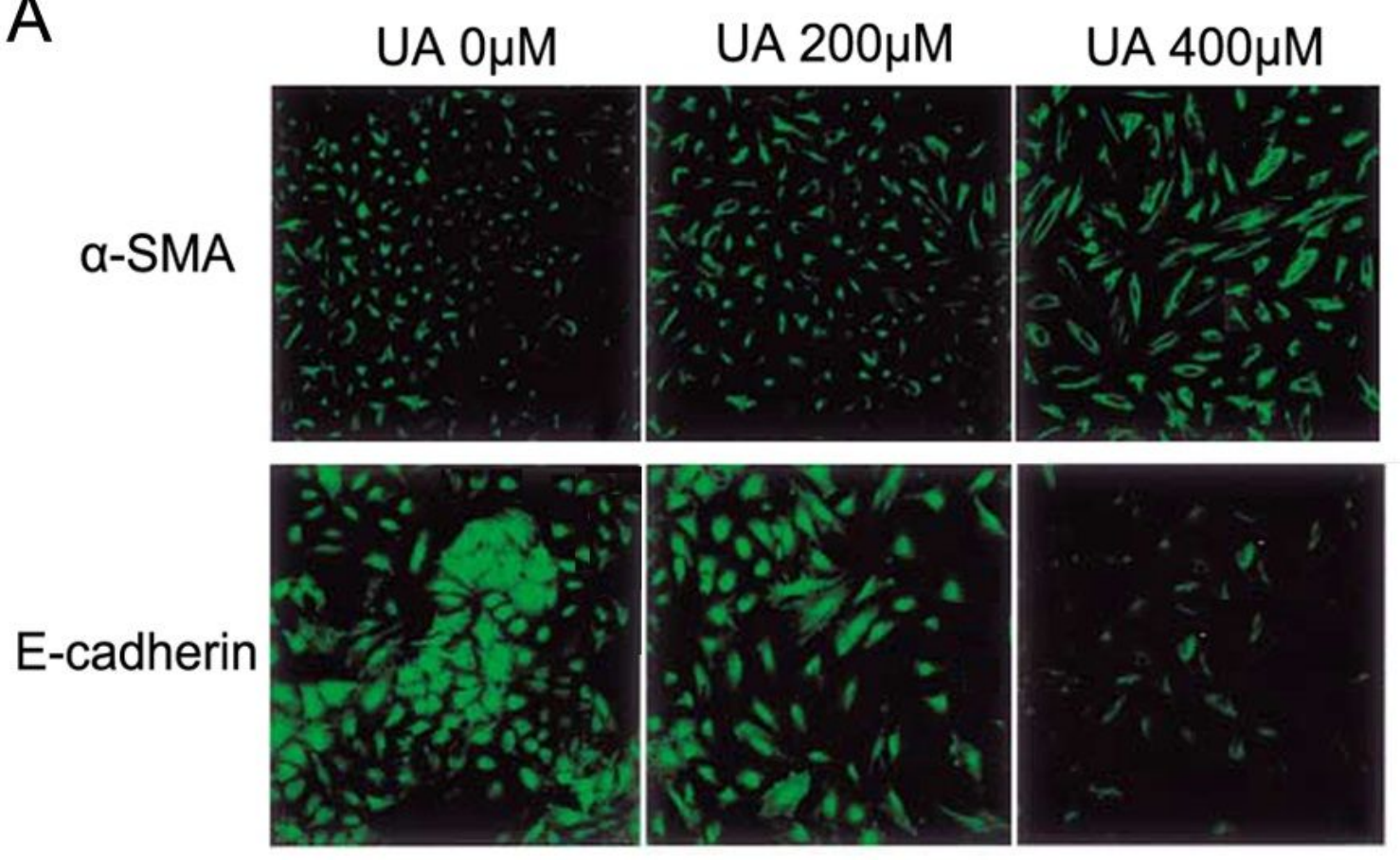

B
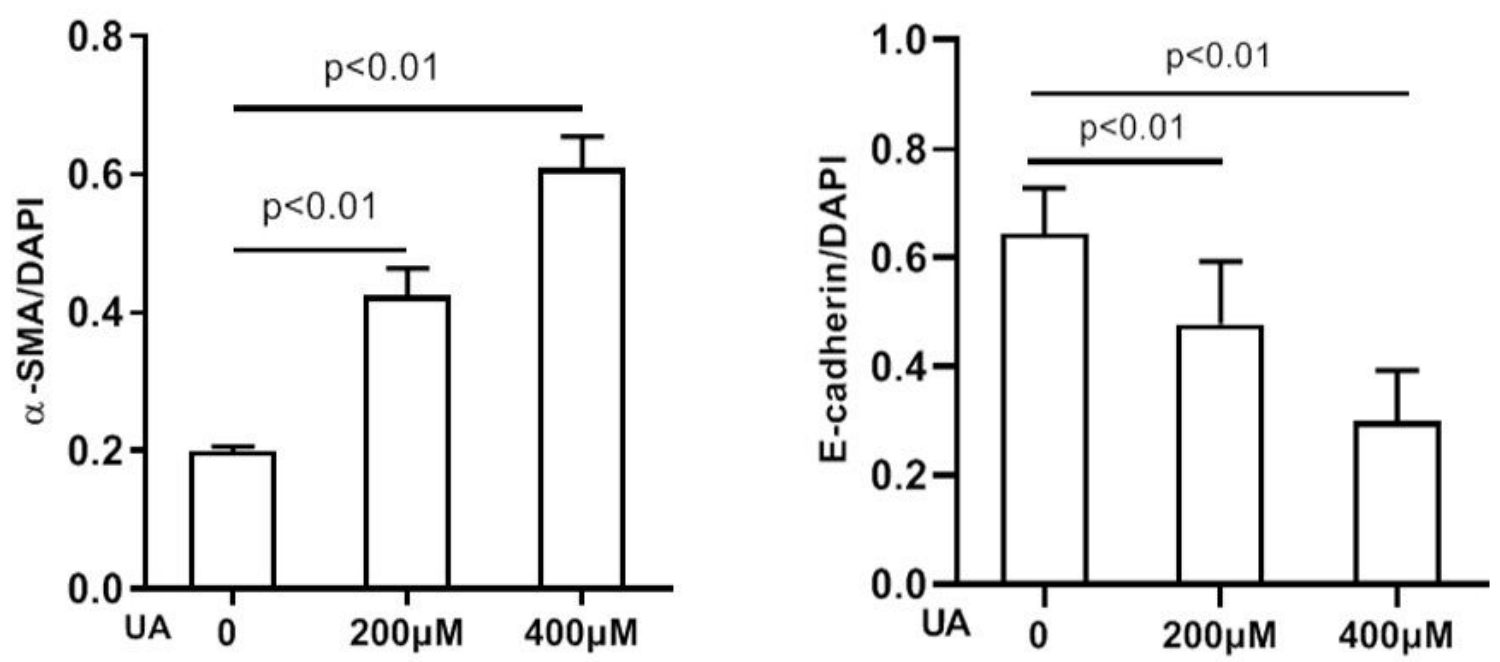

Figure 4

UA induces EMT in primary human lung alveolar type II (A549) cells. UA augmented the expression of the mesenchymal cell marker a-SMA in a dose-dependent manner $(p<0.01)(A, B)$. In contrast, UA decreased the expression of the epithelial cell marker E-cadherin in a dose-dependent manner $(p<0.01)(A, C)$. The fluorescent images were obtained by confocal laser scanning microscope. 
A

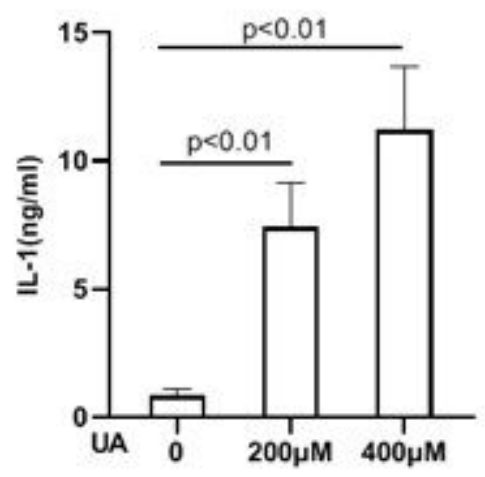

C

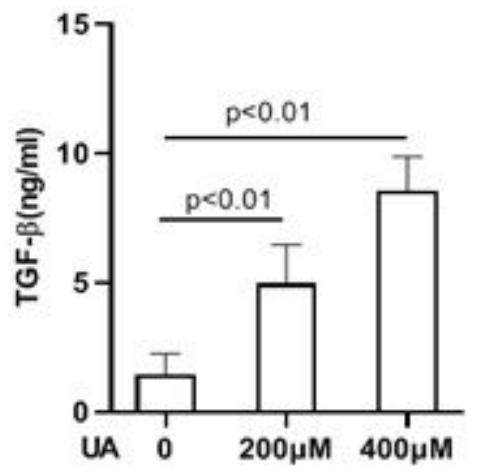

E

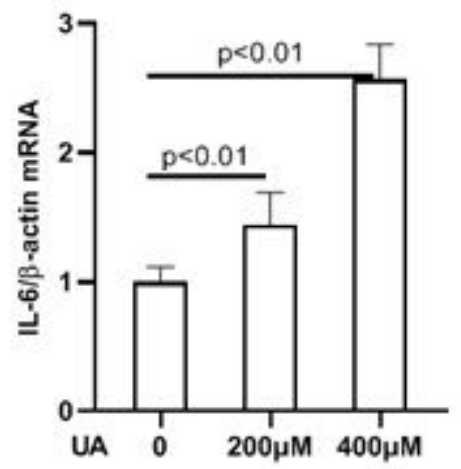

B

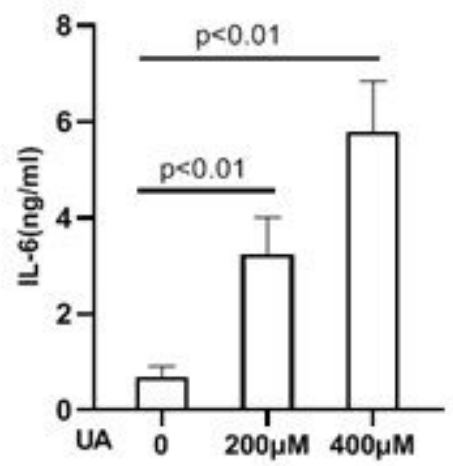

D

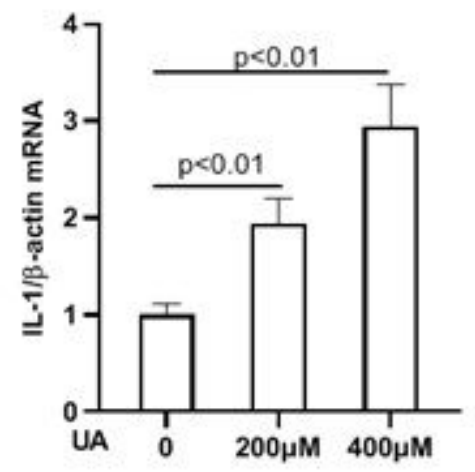

F

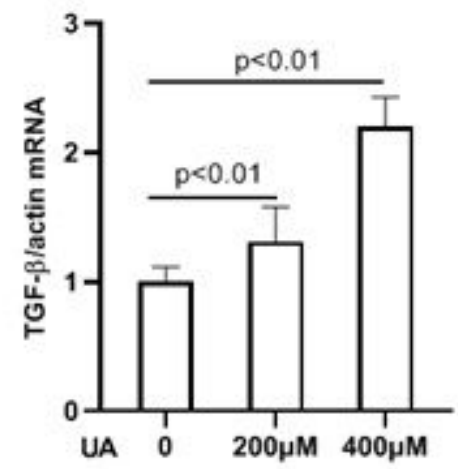

Figure 5

UA induces the production of cytokines in A549 cells. Supernatants of A549 cells stimulated with UA were harvested for ELISA analysis. After $24 \mathrm{~h}$ treatment, the production of IL-1, IL- 6 and transforming growth factor- $\beta$ (TGF- $\beta$ ) by A549 alveolar epithelial cells was markedly increased by UA in a dose-dependent manner compared with that of the control group $(p<0.01)(A, B, C)$. A549 cells treated with increasing concentrations of UA were also harvested for quantitative RT-PCR analysis. mRNA expression of IL-1, IL-6 and TGF- $\beta$ was upregulated by UA in a dose-dependent manner compared with that of the control group $(p<0.01)(D, E, F)$. 